\title{
Design of an Organocatalyst for the Enantioselective Diels-Alder Reaction with $\alpha$-Acyloxyacroleins
}

\author{
Kazuaki Ishihara* and Kazuhiko Nakano \\ Graduate School of Engineering, Nagoya University, Furo-cho, Chikusa-ku, Nagoya 464-8603, Japan
}

General Methods. Infrared (IR) spectra were recorded on a JASCO FT/IR 460 plus spectrometer. $\quad{ }^{1} \mathrm{H}$ NMR spectra were measured on Varian Gemini-2000 (300 MHz) and VXR 500 $(500 \mathrm{MHz})$ spectrometers at ambient temperature. Data were recorded as follows: chemical shift in ppm from internal tetramethylsilane on the $\delta$ scale, multiplicity $(\mathrm{s}=\operatorname{singlet} ; \mathrm{d}=$ doublet; $\mathrm{t}=$ triplet; $\mathrm{q}=$ quartet, $\mathrm{m}=$ multiplet $)$, coupling constant $(\mathrm{Hz})$, integration, and assignment. ${ }^{13} \mathrm{C} \mathrm{NMR}$ spectra were measured on Varian Gemini-2000 (75 MHz) and VXR 500 (125 MHz) spectrometers. Chemical shifts were recorded in ppm from the solvent resonance employed as the internal standard (deuterochloroform at $77.00 \mathrm{ppm}$ ). High performance liquid chromatography (HPLC) analysis was conducted using Shimadzu LC-10 AD coupled diode array-detector SPD-MA-10A-VP and chiral column of Daicel CHIRALCEL OD-H $(4.6 \mathrm{~mm} \times 25 \mathrm{~cm})$ or Daicel CHIRALPAK AD-H (4.6 $\mathrm{mm} \times 25 \mathrm{~cm})$. Optical rotations were measured on a RUDOLPH AUTOPOL IV digital polarimeter. GC analysis was performed with Shimadzu 17A instruments with a flame-ionization detector and a capillary column of PEG-HT Bonded $(25 \mathrm{~m} \times 0.25 \mathrm{~mm})$ using nitrogen as carrier gas. Melting points were determined using a Yanaco MP-J3. The Diels-Alder Reaction was carried out under an atmosphere of air. The others were carried out under an atmosphere of dry nitrogen. For thin-layer chromatography (TLC) analysis throughout this work, Merck precoated TLC plates (silica gel $60 \mathrm{GF} 2540.25 \mathrm{~mm}$ or silica gel $\mathrm{NH}_{2}$ F254S $0.25 \mathrm{~mm}$ ) were used. The products were purified by column chromatography on silica gel (E. Merck Art. 9385 or Fuji Silysia Chemical Ltd. Cromatorex $^{\circledR}$ NH-DM1020). Microanalyses were performed at the Graduate School of Agriculture, Nagoya University. High resolution mass spectral analysis (HRMS) was performed at Chemical Instrument Center, Nagoya University. In experiments that required dry solvent, ether, $N, N$-dimethylformamide (DMF) and tetrahydorofuran (THF) were purchased from TCI or Wako as the "anhydrous" and stored over 4A molecular sieves. Benzene, hexane, toluene, and dichloromethane were freshly distilled from calcium hydride. Other simple chemicals were analytical-grade and obtained commercially.

Ph $\int_{\mathrm{NH}_{2}}^{\mathrm{NH}_{2}}$ 
of $[\mathrm{H} \cdot \mathrm{H}-\mathrm{L}-\mathrm{Phe}-\mathrm{OMe}] \mathrm{Cl}$ in toluene with aqueous ammonium hydroxide gave $\mathrm{H}-\mathrm{L}-\mathrm{Phe}-\mathrm{NH}_{2}$. And then, H-L-Phe- $\mathrm{NH}_{2}$ was reduced with $\mathrm{LiAlH}_{4}$ to give $\mathbf{1 a}$ as a colorless oil in $84 \%$ yield from $[\mathrm{H} \bullet \mathrm{H}-\mathrm{L}-\mathrm{Phe}-\mathrm{OMe}] \mathrm{Cl} . \quad$ IR (film) 3360, 3284, 3026, 2917, 2853, 1602, 1454, 1360, 1072, 1030 $\mathrm{cm}^{-1}$; ${ }^{1} \mathrm{H}$ NMR (300 MHz, $\mathrm{CDCl}_{3}$ ) $\delta 1.24-1.58$ (br, 4H), 2.45-2.58 (m, 2H), 2.75-2.83 (m, 2H),

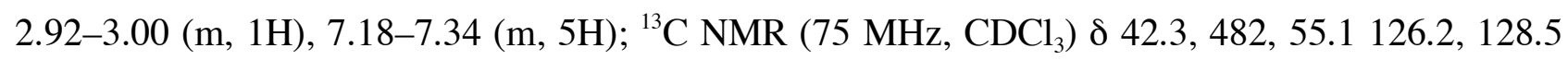
(2C), $129.2(2 \mathrm{C}), 139.2 ;[\alpha]^{20.6}-16.7\left(c 1.10, \mathrm{CHCl}_{3}\right)$.<smiles>N[C@H](CNCc1ccccc1)Cc1ccccc1</smiles>

(+)-(S)-2-Amino-1-( $N$-Benzylamino)-3-phenylpropane $(\mathbf{1 b}){ }^{2} \quad$ According to the literature, ${ }^{3}$ treatment of $[\mathrm{H} \bullet \mathrm{H}-\mathrm{L}-\mathrm{Phe}-\mathrm{OMe}] \mathrm{Cl}$ in toluene with benzylamine gave $\mathrm{H}-\mathrm{L}-\mathrm{Phe}-\mathrm{NHBn}$. And then, H-L-Phe-NHBn was reduced with $\mathrm{LiAlH}_{4}$ to give $\mathbf{1 b}$ as a white solid in $63 \%$ yield from $[\mathrm{H} \bullet \mathrm{H}-\mathrm{L}-\mathrm{Phe}-\mathrm{OMe}] \mathrm{Cl}$. IR (film) 3329, 3084, 3060, 3026, 2918, 1603, 1564, 1495, 1453, 1400, 1263, 1112, $1029 \mathrm{~cm}^{-1} ;{ }^{1} \mathrm{H}$ NMR $\left(\mathrm{CDCl}_{3}\right) \delta$ 1.33-1.56 (br, 3H), 2.49 (dd, $\left.1 \mathrm{H}, J=8.1,11.4 \mathrm{~Hz}\right)$, $2.52(\mathrm{dd}, 1 \mathrm{H}, J=2.1,8.1 \mathrm{~Hz}), 2.74(\mathrm{dd}, 1 \mathrm{H}, J=3.9,11.7 \mathrm{~Hz}), 2.79(\mathrm{dd}, 1 \mathrm{H}, J=4.8,13.5 \mathrm{~Hz}), 3.11$ (tt, $1 \mathrm{H}, J=3.9,7.8 \mathrm{~Hz}), 3.76(\mathrm{~d}, 1 \mathrm{H}, J=13.5 \mathrm{~Hz}), 3.82(\mathrm{~d}, 1 \mathrm{H}, J=13.2 \mathrm{~Hz}), 7.10-7.39(\mathrm{~m}, 10 \mathrm{H})$; ${ }^{13} \mathrm{C}$ NMR $\left(75 \mathrm{MHz}, \mathrm{CDCl}_{3}\right) \delta 42.8,52.6,54.1,55.5,126.2,126.9,128.1$ (2C), $128.3(2 \mathrm{C}), 128.4$ (2C), $129.2(2 \mathrm{C}), 139.2,140.5 ;[\alpha]^{20.2}=15.3\left(c 1.87, \mathrm{CHCl}_{3}\right)$.<smiles>CC(C)C(N)CNCc1ccccc1</smiles>

(+)-(S)-2-Amino-1-(N-benzylamino)-3-methylbutane (1e). ${ }^{4}$ According to the literature, ${ }^{3}$ treatment of $[\mathrm{H} \bullet \mathrm{H}-\mathrm{L}-\mathrm{Val}-\mathrm{OMe}] \mathrm{Cl}$ in toluene with benzylamine gave $\mathrm{H}-\mathrm{L}-\mathrm{Phe}-\mathrm{NHBn}$ in high yield. And then, H-L-Phe-NHBn was reduced with $\mathrm{LiAlH}_{4}$ to give $1 \mathrm{e}$ as a colorless oil in high yield. IR (film) 3306, 3027, 2957, 2871, 1495, 1454, 1365, 1115, $1028 \mathrm{~cm}^{-1 ; 1} \mathrm{H}$ NMR (300 $\left.\mathrm{MHz}, \mathrm{CDCl}_{3}\right) \delta 0.88(\mathrm{~d}, 3 \mathrm{H}, J=4.8 \mathrm{~Hz}), 0.91(\mathrm{~d}, 3 \mathrm{H}, J=4.8 \mathrm{~Hz}), 1.39$ (br, 3H), 1.59 (septet, $1 \mathrm{H}, J$ $=4.8 \mathrm{~Hz}), 2.39(\mathrm{dd}, 1 \mathrm{H}, J=9.6,11.4 \mathrm{~Hz}), 2.70(\mathrm{~m}, 1 \mathrm{H}), 2.72(\mathrm{dd}, 1 \mathrm{H}, J=3.3,11.4 \mathrm{~Hz}), 3.80(\mathrm{dd}$, $1 \mathrm{H}, J=13.2,20.7 \mathrm{~Hz}), 7.24-7.33(\mathrm{~m}, 5 \mathrm{H}) ;{ }^{13} \mathrm{C} \mathrm{NMR}\left(125 \mathrm{MHz}, \mathrm{CDCl}_{3}\right) \delta 17.8,19.4,32.3,53.6$, $54.1,56.5,126.8,128.0(2 \mathrm{C}), 128.3(2 \mathrm{C}), 140.6 ;[\alpha]_{\mathrm{D}}^{25.4}=33.4\left(c 1.80, \mathrm{CHCl}_{3}\right)$.<smiles>CCNCC(Cc1ccccc1)NCC</smiles>

(+)-(S)-1,2-Di( $N$-ethylamino)-3-phenylpropane $\quad(N$-Et-1f): $\quad N$-Et-1f $\quad$ was synthesized as a colorless oil in high yield by the acetylation of $\mathbf{1 a}$ with acetic anhydride and the subsequent reduction of the corresponding diacetamide with $\mathrm{LiAlH}_{4}$. IR (film) 3300, 3206, 2963, 2816, 1602, 1495, 1454, 1379, 1121, $1030 \mathrm{~cm}^{-1}$; ${ }^{1} \mathrm{H}$ NMR $\left(500 \mathrm{MHz}, \mathrm{CDCl}_{3}\right) \delta 1.07$ (t, 6H, $J=7.5$ $\mathrm{Hz}), 1.43$ (br, 2H), 2.46 (dd, $J=7.5,12.0 \mathrm{~Hz}), 2.53-2.74(\mathrm{~m}, 6 \mathrm{H}), 2.78-2.89(\mathrm{~m}, 2 \mathrm{H}), 7.18-7.30(\mathrm{~m}$, $5 \mathrm{H}) ;{ }^{13} \mathrm{C}$ NMR $\left(125 \mathrm{MHz}, \mathrm{CDCl}_{3}\right) \delta 30.3,34.2,39.6,41.6,44.3,52.9,59.1,126.1,128.3$ (2C), 
$129.2(2 \mathrm{C}), 139.4 ;[\alpha]^{21.3}=24.3\left(c 1.55, \mathrm{CHCl}_{3}\right)$.

Preparation of Chiral Diamines and Triamines 1c,d,g-j Derived from Boc-L-amino Acids (Boc-Xxx-OH)

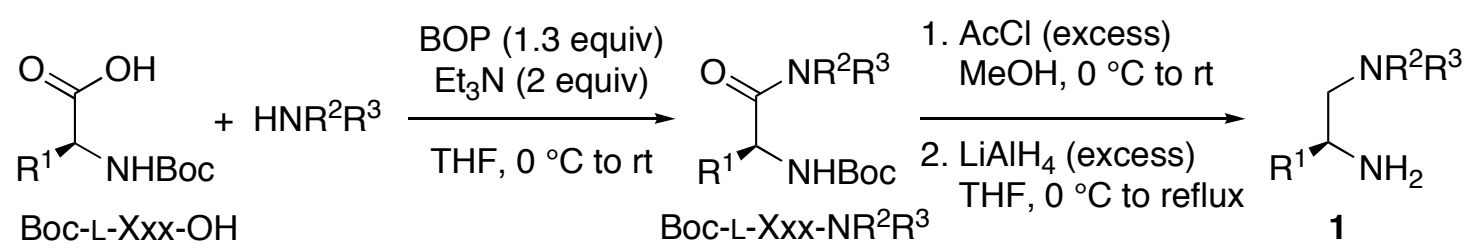

General Procedure for Preparating Monopeptides Boc-L-Xxx-NR ${ }^{2} \mathbf{R}^{3}$. To a solution of Boc-L-Xxx-OH (1.33 g, $5 \mathrm{mmol})$, triethylamine $(1.39 \mathrm{~mL}, 10 \mathrm{mmol})$, and a primary or secondary amine $\quad(5 \quad \mathrm{mmol}) \quad$ in $\quad \mathrm{THF} \quad(20 \quad \mathrm{~mL}) \quad$ was added (benzotriazol-1-yloxy)tris(dimethylamino)phosphonium hexafluorophosphate (BOP, $2.87 \mathrm{~g}, 6.5$ mmol) at $0{ }^{\circ} \mathrm{C}$. After being stirred for $2 \mathrm{~h}$ at the same temperature, the reaction mixture was allowed to warm to ambient temperature and stirred for an additional $6 \mathrm{~h}$. The reaction was quenched with water, and the solution was concentrated in vacuo. The residue was extracted with EtOAc or $\mathrm{CHCl}_{3}$ and washed with saturated aqueous $\mathrm{NaHCO}_{3}$ and brine. The organic layer was dried over $\mathrm{Na}_{2} \mathrm{SO}_{4}$, filtered, and concentrated in vacuo. The crude product was purified by column chromatography on silica gel using hexane-EtOAc or $\mathrm{CHCl}_{3}-\mathrm{MeOH}$ as eluent to give the corresponding monopeptide Boc-L-Xxx-NR ${ }^{2} \mathrm{R}^{3}$.<smiles>COC(=O)N[C@H](Cc1ccccc1)C(=O)N1CCCC1</smiles>
9H), 1.48-1.86 (m, 4H), 2.51-2.59 (m, 1H), 2.84-3.06 (m, 2H), 3.22-3.48 (m, 3H), 4.58 (dd, 1H, $J$ $=7.2,13.2 \mathrm{~Hz}), 5.41(\mathrm{~d}, 1 \mathrm{H}, J=8.4 \mathrm{~Hz}), 7.20-7.29(\mathrm{~m}, 5 \mathrm{H})$.<smiles>CCCN(C(=O)O)C(=O)C(Cc1ccccc1)NC(=O)O</smiles>
$(\mathrm{d}, 3 \mathrm{H}, J=6.9 \mathrm{~Hz}), 0.97$ (d, 3H, $J=6.9 \mathrm{~Hz}), 1.42$ (s, 9H), 2.07-2.33 (br, 1H), 3.92 (dd, 1H, $J=6.3$, $8.7 \mathrm{~Hz}), 4.5(\mathrm{t}, 1 \mathrm{H}, J=7.8 \mathrm{~Hz}), 5.86(\mathrm{br}, 1 \mathrm{H}), 7.21-7.35(\mathrm{~m}, 5 \mathrm{H})$.<smiles>CCCNC(=O)[C@H](Cc1ccccc1)NCCOC(C)(C)C</smiles>

Boc-L-Phe-NH( $\left(\mathrm{CH}_{2}\right)_{2} \mathrm{NMe}_{2}: \quad 93 \%$ yield (white solid); ${ }^{1} \mathrm{H}$ NMR $\left(\mathrm{CDCl}_{3}\right) \delta$ $1.41(\mathrm{~s}, 9 \mathrm{H}), 2.07(\mathrm{~s}, 6 \mathrm{H}), 2.23(\mathrm{t}, 2 \mathrm{H}, J=6.0 \mathrm{~Hz}), 3.01(\mathrm{dd}, 1 \mathrm{H}, J=7.2,13.2 \mathrm{~Hz}), 3.09(\mathrm{dd}, 1 \mathrm{H}, J=$ 6.0, 13.2), 3.26 (br, 2H), 4.26 (br, $1 \mathrm{H}), 5.01(\mathrm{br}, 1 \mathrm{H}), 7.18-7.32(\mathrm{~m}, 5 \mathrm{H})$. 
<smiles>CN(C)CCCNC(=O)C(Cc1ccccc1)NC(C)(C)C</smiles>

Boc-L-Phe-NH( $\left(\mathrm{CH}_{2}\right)_{3} \mathbf{N M e}_{2}$ : 97\% yield (white solid); ${ }^{1} \mathrm{H}$ NMR (300 $\left.\mathrm{MHz}, \mathrm{CDCl}_{3}\right) \delta 1.40(\mathrm{~s}, 9 \mathrm{H}), 1.44-1.59(\mathrm{br}, 2 \mathrm{H}), 2.01(\mathrm{~s}, 6 \mathrm{H}), 2.16-2.28(\mathrm{~m}, 2 \mathrm{H}), 3.05(\mathrm{t}, 2 \mathrm{H}, J=$ $6.5 \mathrm{~Hz}$ ), 3.17-3.34 (br, 2H), 4.18-4.36 (br, 1H), 5.04-5.20 (br, 1H), 7.13-7.38 (m, 5H).

General Procedure for Preparing Diamines and Triamines 1 from Boc-L-Xxx-NR ${ }^{2} R^{3}$ : To a solution of Boc-L-Xxx-NR ${ }^{2} \mathrm{R}^{3}(4.5 \mathrm{mmol})$ in $\mathrm{MeOH}(45 \mathrm{~mL})$ was added dropwise acetyl chloride $(4.5 \mathrm{~mL})$ at $0{ }^{\circ} \mathrm{C}$. After being stirred for $3 \mathrm{~h}$ at $0{ }^{\circ} \mathrm{C}$, the reaction was allowed to warm to ambient temperature and stirred for an additional $1 \mathrm{~h}$ and the solution was concentrated in vacuo. To the residual product, $\mathrm{H}-\mathrm{L}-\mathrm{Xxx}-\mathrm{NR}^{2} \mathrm{R}^{3}$, in THF $(50 \mathrm{~mL})$ was added lithium aluminum hydride (120 $\mathrm{mg}$, $31.5 \mathrm{mmol}$ ) at $0{ }^{\circ} \mathrm{C}$. After being stirred for $30 \mathrm{~min}$ at $0{ }^{\circ} \mathrm{C}$, the reaction was allowed to heat at reflux for $48 \mathrm{~h}$ before the reaction was quenched with $\mathrm{Na}_{2} \mathrm{SO}_{4}(1.39 \mathrm{~g})$ and water $(3.62 \mathrm{~mL})$ with vigorous stirring at $0{ }^{\circ} \mathrm{C}$. The white-gray suspension was filtered and concentrated. The crude product was purified by column chromatography on Cromatorex ${ }^{\circledR}$ NH-DM1020 (eluent: hexane-EtOAc) to give the corresponding diamine or triamine $\mathbf{1}$.<smiles>NC(Cc1ccccc1)CN1CCCC1</smiles>

(+)-(S)-2-Amino-1-phenyl-3-(pyrrolidin-1-yl)propane $\quad(\mathbf{1 c}):^{5} \quad 86 \%$ yield (colorless oil); IR (film) 3060, 3026, 2964, 2927, 2874, 2783, 2359, 1602, 1495, 1454, 1357, 1146, 1076, $1031 \mathrm{~cm}^{-1} ;{ }^{1} \mathrm{H}$ NMR $\left(\mathrm{CDCl}_{3}\right) \delta 1.71(\mathrm{br}, 2 \mathrm{H}), 1.71-1.80(\mathrm{~m}, 4 \mathrm{H}), 2.31(\mathrm{dd}, 1 \mathrm{H}, J=4.2,11.7$ $\mathrm{Hz}), 2.40-2.65(\mathrm{~m}, 6 \mathrm{H}) 2.78(\mathrm{dd}, 1 \mathrm{H}, J=4.5,13.2 \mathrm{~Hz}), 3.15(\mathrm{tt}, 1 \mathrm{H}, J=4.2,8.4 \mathrm{~Hz}), 7.20-7.32(\mathrm{~m}$, $5 \mathrm{H}) ;{ }^{13} \mathrm{C} \mathrm{NMR}\left(125 \mathrm{MHz}, \mathrm{CDCl}_{3}\right) \delta 23.5$ (2C), 42.6, 51.3, 54.4 (2C), 63.2, 126.1, 128.4 (2C), 129.3 (2C), 139.5; $[\alpha]^{27}=16.2\left(c 1.11, \mathrm{CHCl}_{3}\right)$.<smiles>CCCN(CCC)CC(N)Cc1ccccc1</smiles>

(+)-(S)-2-Amino-1-( $\boldsymbol{N}, \boldsymbol{N}$-diisopropylamino)-3-phenylpropane (1d): $71 \%$ yield (colorless oil); bp. 80 100 ${ }^{\circ} \mathrm{C}$ (40 Pa); IR (film) 3062, 3027, 2964, 1602, 1495, 1454, 1362, 1206, 1161, 1119, 1081, $1055 \mathrm{~cm}^{-1}$; ${ }^{1} \mathrm{H}$ NMR $\left(300 \mathrm{MHz}, \mathrm{CDCl}_{3}\right) \delta 0.97(\mathrm{~d}, 3 \mathrm{H}, J=6.6 \mathrm{~Hz}), 1.02(\mathrm{~d}, 3 \mathrm{H}, J$ $=6.6 \mathrm{~Hz}), 1.36-1.50(\mathrm{br}, 2 \mathrm{H}), 2.25(\mathrm{dd}, 1 \mathrm{H}, J=6.6,12.6 \mathrm{~Hz}), 2.39(\mathrm{dd}, 1 \mathrm{H}, J=9.0,13.5 \mathrm{~Hz}), 2.53$ (dd, 1H, $J=3.9,13.5 \mathrm{~Hz}), 2.77(\mathrm{dd}, 1 \mathrm{H}, J=3.9,13.2 \mathrm{~Hz}), 2.93-3.13(\mathrm{~m}, 3 \mathrm{H}), 7.17-7.40(\mathrm{~m}, 5 \mathrm{H})$; ${ }^{13} \mathrm{C} \mathrm{NMR}\left(125 \mathrm{MHz}, \mathrm{CDCl}_{3}\right.$ ) $\delta 19.7$ (2C), 22.2 (2C), 42.3, 47.8 (2C), 51.1, 51.7, 125.9, 128.3 (2C), $129.1(2 \mathrm{C}), 140.1 ;[\alpha]_{\mathrm{D}}^{20.8}=0.8\left(c 2.0, \mathrm{CHCl}_{3}\right)$.<smiles>CN(C)CCNCC(N)Cc1ccccc1</smiles>

(+)-(S)-2-Amino-1-\{N-[2-(dimethylamino)ethyl]amino\}-3-phenylpropane 
(1g): $70 \%$ yield (colorless oil); bp. $110-130{ }^{\circ} \mathrm{C}(1 \mathrm{~Pa})$; IR (film) 3298, 3060, 2939, 2817, 1602 , 1496, 1455, 1360, 1270, 1134, $1040 \mathrm{~cm}^{-1} ;{ }^{1} \mathrm{H}$ NMR (300 MHz, $\mathrm{CDCl}_{3}$ ) $\delta 2.22$ (s, 6H) 2.32-2.45 (m, 2H), 2.45-2.55 (m, 2H), 2.60-2.83 (m, 4H), 3.05-3.16 (m, 1H), 7.19-7.31 (m, 5H); ${ }^{13} \mathrm{C}$ NMR $(75$ $\left.\mathrm{MHz}, \mathrm{CDCl}_{3}\right) \delta 42.7,45.5(2 \mathrm{C}), 47.5,52.5,56.4,59.6,126.1,128.4(2 \mathrm{C}), 129.2(2 \mathrm{C}), 139.2 ;[\alpha]^{20.2}$ $=2.5\left(c 1.47, \mathrm{CHCl}_{3}\right)$.<smiles>CN(C)CCCNCC(N)Cc1ccccc1</smiles>

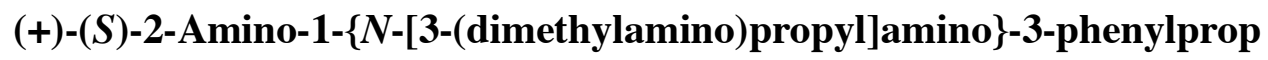
ane (1h): Bp. $120-135{ }^{\circ} \mathrm{C}(1 \mathrm{~Pa}) ; 53 \%$ yield (colorless oil); ${ }^{1} \mathrm{H} \mathrm{NMR}\left(300 \mathrm{MHz}, \mathrm{CDCl}_{3}\right) \delta$ 1.40-1.80 (br, 3H), 1.65 (quintet, $2 \mathrm{H}, J=6.9 \mathrm{~Hz}$ ), 2.21 (s, 6H), 2.31 (t, 2H, $J=7.2 \mathrm{~Hz}$ ), 2.43-2.54 $(\mathrm{m}, 2 \mathrm{H}), 2.56-2.66(\mathrm{~m}, 2 \mathrm{H}), 2.71(\mathrm{dd}, 1 \mathrm{H}, J=3.9,12.0 \mathrm{~Hz}), 2.78(\mathrm{dd}, 1 \mathrm{H}, J=4.8,13.5 \mathrm{~Hz}), 3.10$ (tt, $J=3.6,7.2 \mathrm{~Hz}), 7.19-7.36(\mathrm{~m}, 5 \mathrm{H})$.<smiles>CNCCNCC(N)Cc1ccccc1</smiles>

(+)-(S)-2-Amino-1-\{N-[2-(methylamino)ethyl]amino\}-3-phenylpropane

(1i): $56 \%$ yield (colorless oil); IR (film) 3296, 3026, 2928, 2840, 2360, 1602, 1495, 1454, 1359, 1111, $1031 \mathrm{~cm}^{-1}$; ${ }^{1} \mathrm{H}$ NMR $\left(300 \mathrm{MHz}, \mathrm{CDCl}_{3}\right.$ ) $\delta$ 1.21-1.60 (br, 4H), 2.43 (s, 3H), 2.41-2.54 (m, $2 \mathrm{H}), 2.63-2.83(\mathrm{~m}, 6 \mathrm{H}), 3.10(\mathrm{tt}, 1 \mathrm{H}, J=4.2,8.4 \mathrm{~Hz}), 7.18-7.35(\mathrm{~m}, 5 \mathrm{H}) ;{ }^{13} \mathrm{C} \mathrm{NMR}(75 \mathrm{MHz}$, $\left.\mathrm{CDCl}_{3}\right) \delta 36.5,42.8,49.4,51.8,52.6,56.2,126.2,128.4(2 \mathrm{C}), 129.2(2 \mathrm{C}), 139.2 ;[\alpha]_{\mathrm{D}}^{21.6}=5.11(c$ $\left.1.27, \mathrm{CHCl}_{3}\right)$.<smiles>CN(C)CC(N)Cc1ccccc1</smiles>

(+)-(S)-2-Amino-1-\{N-[2-(dimethylamino)ethyl]- $N$-methylamino\}-3-phen yl-propane (1j): $\quad 62 \%$ yield (colorless oil); IR (film) 3370, 3297, 3026, 2941, 1602, 1496, 1357 , 1122, $1031 \mathrm{~cm}^{-1} ;{ }^{1} \mathrm{H}$ NMR (300 MHz, $\left.\mathrm{CDCl}_{3}\right) \delta 2.24(\mathrm{~s}, 6 \mathrm{H}), 2.25$ (s, 3H), 2.29-2.31 (m, 2H), 2.35-2.60 (m, 4H), $2.46(\mathrm{dd}, 1 \mathrm{H}, J=8.7,13.2 \mathrm{~Hz}), 2.72(\mathrm{dd}, J=4.8,12.9 \mathrm{~Hz}), 3.10-3.19(\mathrm{~m}, 1 \mathrm{H})$, 7.19-7.31 (m, 5H); ${ }^{13} \mathrm{C}$ NMR (75 MHz, $\left.\mathrm{CDCl}_{3}\right) \delta$ 42.3, 43.0, 45.9 (2C), 50.0, 56.4, 57.8, 65.0, $126.1,128.4(2 \mathrm{C}), 129.2(2 \mathrm{C}), 139.4 ;[\alpha]^{20.7}=27.7\left(c 1.81, \mathrm{CHCl}_{3}\right)$.

General Procedure for Preparing Chiral Triamines Derived from Dipeptides: Solutionand solid-phase synthetic methods of dipeptides are shown below.

Solution-Phase Synthesis of Dipeptides. 

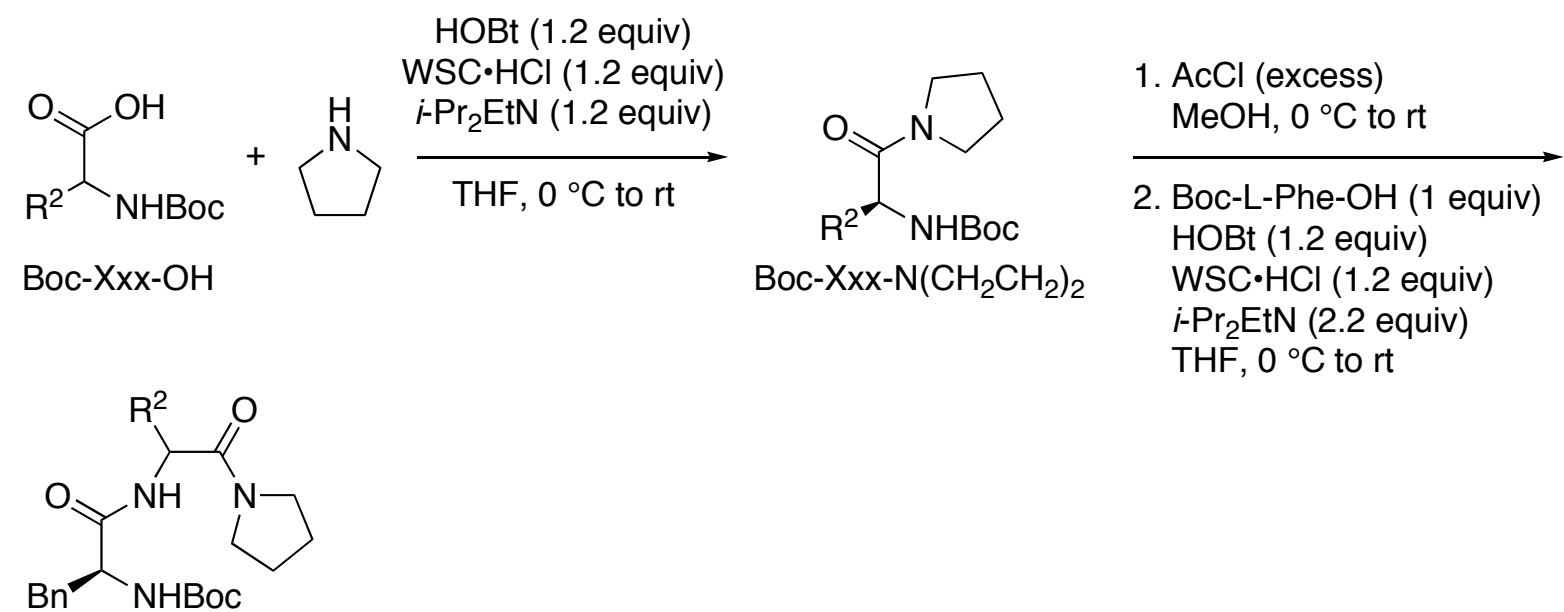

Boc-L-Phe-Xxx-N $\left(\mathrm{CH}_{2} \mathrm{CH}_{2}\right)_{2}$

General Procedure for Preparing Monopeptides Boc-Xxx-N( $\left(\mathrm{CH}_{2} \mathrm{CH}_{2}\right)_{2}$ : To a solution of $\mathrm{N}$-Boc-amino acid (Boc-Xxx-OH, $5 \mathrm{mmol}$ ), 1-hydroxybenzotriazole (HOBt, $811 \mathrm{mg}, 6 \mathrm{mmol}$ ), 1-ethyl-3-(3'-dimethylaminopropyl)carbodiimide hydrochloride (WSC•HCl, $1.15 \mathrm{~g}, 6 \mathrm{mmol}$ ), and $i$-Pr ${ }_{2} \mathrm{NEt}(1.05 \mathrm{~mL}, 6 \mathrm{mmol})$ in THF $(15 \mathrm{~mL})$ was added pyrrolidine $(0.500 \mathrm{~mL}, 6 \mathrm{mmol})$ at $0{ }^{\circ} \mathrm{C}$. After being stirred at the same temperature for $0.5 \mathrm{~h}$, the reaction was allowed to warm to ambient temperature and stirred for an additional $12 \mathrm{~h}$. The reaction was quenched with $\mathrm{H}_{2} \mathrm{O}$. The reaction mixture was extracted with EtOAc and washed with brine and $1 \mathrm{M} \mathrm{HCl}$ aqueous solution. The organic layer was dried over $\mathrm{Na}_{2} \mathrm{SO}_{4}$, filtered, and concentrated. The crude product was purified by column chromatography on silica gel using hexane-EtOAc or $\mathrm{CHCl}_{3}-\mathrm{MeOH}$ as eluent to give Boc- $\mathrm{Xxx}-\mathrm{N}\left(\mathrm{CH}_{2} \mathrm{CH}_{2}\right)_{2}$.

General Procedure for Preparing Dipeptides Boc-L-Phe-Xxx-N( $\left(\mathrm{CH}_{2} \mathrm{CH}_{2}\right)_{2}$ : To a solution of monopeptide Boc-Xxx-N( $\left(\mathrm{CH}_{2} \mathrm{CH}_{2}\right)_{2}(5 \mathrm{mmol})$ in $\mathrm{MeOH}(50 \mathrm{~mL})$ was added dropwise acetyl chloride $(5 \mathrm{~mL})$ at $0{ }^{\circ} \mathrm{C}$. After being stirred for $3 \mathrm{~h}$ at $0{ }^{\circ} \mathrm{C}$, the reaction was allowed to warm to ambient temperature and stirred for an additional $1 \mathrm{~h}$ and the solution was concentrated in vacuo to give $\mathrm{H}-\mathrm{L}-\mathrm{Xxx}-\mathrm{N}\left(\mathrm{CH}_{2} \mathrm{CH}_{2}\right)_{2}$. To a solution of $\mathrm{H}-\mathrm{Xxx}-\mathrm{N}\left(\mathrm{CH}_{2} \mathrm{CH}_{2}\right)_{2}(5 \mathrm{mmol})$, Boc-L-Phe-OH (1.33 g, $5 \mathrm{mmol})$, HOBt $(811 \mathrm{mg}, 6 \mathrm{mmol})$, and WSC $\cdot \mathrm{HCl}(1.15 \mathrm{~g}, 6 \mathrm{mmol})$ in THF (20mL) was added $i$-Pr ${ }_{2} \mathrm{NEt}(1.92 \mathrm{~mL}, 11 \mathrm{mmol})$ at $0{ }^{\circ} \mathrm{C}$. After being stirred at the same temperature for $0.5 \mathrm{~h}$, the reaction was allowed to warm to ambient temperature and stirred for an additional $12 \mathrm{~h}$. The reaction was quenched with $\mathrm{H}_{2} \mathrm{O}$. The reaction mixture was extracted with EtOAc and washed with brine and $1 \mathrm{M} \mathrm{HCl}$ aqueous solution. The organic layer was dried over $\mathrm{Na}_{2} \mathrm{SO}_{4}$, filtered, and concentrated. The crude product was purified by column chromatography on silica gel using hexane-EtOAc or $\mathrm{CHCl}_{3}-\mathrm{MeOH}$ as eluent to give Boc-L-Phe-Xxx-N( $\left.\mathrm{CH}_{2} \mathrm{CH}_{2}\right)_{2}$.

\section{Solid-Phase Synthesis of Dipeptides.}




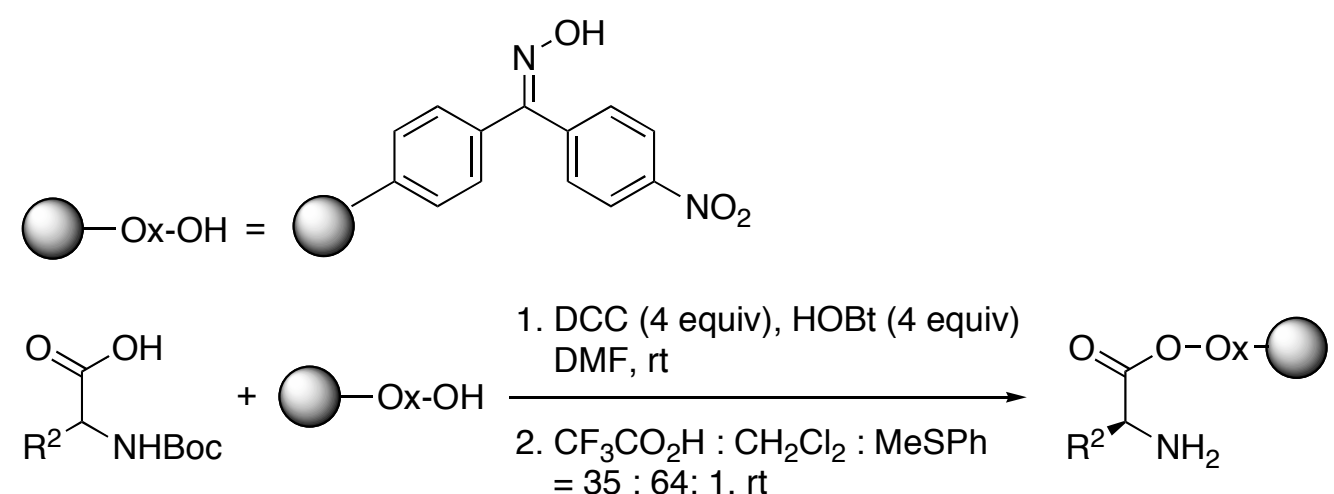

Boc-Xxx-OH

(4 equiv)

$\mathrm{H}-\mathrm{Xxx}-\mathrm{O}$-oxime resin

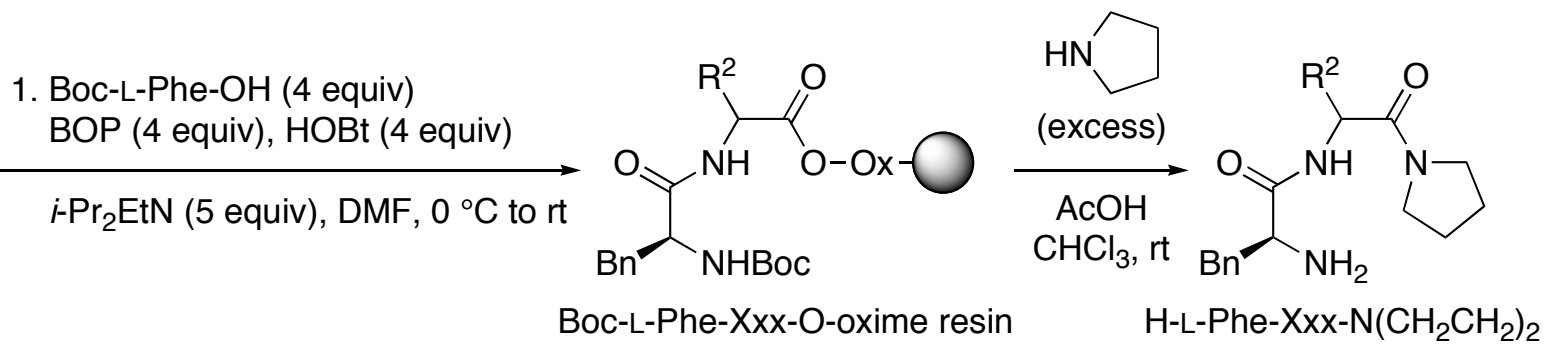

Manual solid-phase peptide synthesis was carried out using oxime resin purchased from Wako having a loading of approximately $0.6 \mathrm{mmol} / \mathrm{g} .^{6} \quad$ Dichloromethane (DCM), isopropanol (IPA), and $N, N$-dimethylformamide (DMF) used were reagent grade. DMF was stored over $4 \AA$ molecular sieves to reduce the amount of primary and secondary amines present in the solvent. The TFA-deprotecting solution (TFA : DCM : thioanisole = $35: 64: 1$ ) was stored under dry nitrogen and protected from light. The oxime resin $(0.6 \mathrm{mmol}$ of oxime unit $/ \mathrm{g}, 1 \mathrm{~g})$ purchased was washed in a synthesis vessel with DCM $(1 \times 1 \mathrm{~min})$, IPA $(1 \times 1 \mathrm{~min})$ and DCM $(2 \times 1 \mathrm{~min})$. The first Boc-amino acid, Boc-Xxx-OH, was loaded onto the resin by shaking a mixture of dicyclohexylcarbodiimide (DCC, 4 equiv), HOBt (4 equiv), Boc-Xxx-OH (3 equiv) with the oxime resin for $24 \mathrm{~h}$ in DMF ( $4 \mathrm{~mL})$. The Boc-Xxx-O-oxime resin was then washed with DCM $(2 \times 1$ min), IPA $(2 \times 1 \mathrm{~min}), \mathrm{DCM}(1 \times 1 \mathrm{~min}), \mathrm{IPA}(1 \times 1 \mathrm{~min})$, and DCM $(3 \times 1 \mathrm{~min})$.

The Boc-Xxx-O-oxime resin was deprotected with TFA $(35 \%$ TFA $\times 30 \mathrm{~min})$ to transform the trifluoroacetate ammonium form, and washed with DCM $(2 \times 1 \mathrm{~min})$, IPA $(2 \times 1 \mathrm{~min})$, DCM $(1 \times 1$ min), IPA $(1 \times 1 \mathrm{~min}), \operatorname{DCM}(1 \times 1 \mathrm{~min})$, and DMF $(2 \times 1 \mathrm{~min})$.

Next, $N, N$-diisopropylethylamine (5 equiv) was added a pre-cooled solution of the second Boc-amino acid (Boc-L-Phe-OH, 4 equiv) and BOP (4 equiv) in DMF (4 mL), and the mixture was stirred at ambient temperature for 10 15 min to preactivate Boc-L-Phe-OH. After that, the preactivated solution of Boc-L-Phe-OH was added to the trifluoroacetate ammonium form of the resin. After being shaken for $3 \mathrm{~h}$, the resin was washed with DCM $(2 \times 1 \mathrm{~min})$, IPA $(2 \times 1 \mathrm{~min})$, DCM $(1 \times 1 \mathrm{~min})$, IPA $(1 \times 1 \mathrm{~min})$, DCM $(3 \times 1 \mathrm{~min})$, and dried under vacuum.

Each steps were monitored by the Kaiser ninhydrin test. 


\section{General Procedure for the Nucleophilic Cleavage of Dipeptides}

Boc-L-Phe-Xxx-N $\left(\mathrm{CH}_{2} \mathrm{CH}_{2}\right)_{2}$ from the Oxime Resin: To a $10 \mathrm{~mL}$ of peptide synthesis vessel was added Boc-L-Phe-Xxx-oxime resin $(1 \mathrm{~g})$. The resin was swollen in $\mathrm{CHCl}_{3}$ and was pyrrolidine $(334 \mu \mathrm{L}, 0.8 \mathrm{mmol})$ and acetic acid $(228 \mu \mathrm{L}, 4 \mathrm{mmol})$ added, and the contents were agitated for $6 \mathrm{~h}$. The resulting solution was drained into a sidearm filtration flask and the resin washed with $\mathrm{CHCl}_{3}(2 \times 5 \mathrm{~mL}), 1: 1 \mathrm{CHCl}_{3} / \mathrm{DMF}(2 \times 5 \mathrm{~mL})$, and $\mathrm{CHCl}_{3}(2 \times 5 \mathrm{~mL})$, collecting all of the washes. The combined washes were concentrated under reduced pressure to $5 \sim 10 \mathrm{~mL}$, and the solution of crude peptide was extracted with $\mathrm{CHCl}_{3}$ and washed with saturated aqueous $\mathrm{NaHCO}_{3}$ and brine. The organic layer was dried over $\mathrm{Na}_{2} \mathrm{SO}_{4}$, filtered, and concentrated. The crude product was purified by column chromatography on silica gel using as $\mathrm{CHCl}_{3}-\mathrm{MeOH}$ eluent to give Boc-L-Phe- $\mathrm{Xxx}-\mathrm{N}\left(\mathrm{CH}_{2} \mathrm{CH}_{2}\right)_{2}$.

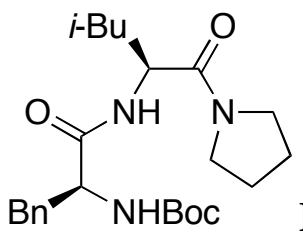

Boc-L-Phe-L-Leu-N $\left(\mathrm{CH}_{2} \mathbf{C H}_{2}\right)_{2}$ : $97 \%$ yield (white solid) from Boc-L-Leu-OH (liquid-phase synthesis); ${ }^{1} \mathrm{H}$ NMR $\left(300 \mathrm{MHz}, \mathrm{CDCl}_{3}\right) \delta 0.89(\mathrm{~d}, 3 \mathrm{H}, J=6.3 \mathrm{~Hz}), 0.97(\mathrm{~d}, 3 \mathrm{H}, J=$ $6.3 \mathrm{~Hz}), 1.33-1.64(\mathrm{~m}, 3 \mathrm{H}), 1.40(\mathrm{~s}, 9 \mathrm{H}), 1.80-2.02(\mathrm{~m}, 4 \mathrm{H}), 3.07$ (d, 2H, 6.0 Hz), 3.34-3.52 (m, 3H), 3.51-3.66 (m, 1H), $4.38(\mathrm{br}, 1 \mathrm{H}), 4.74(\mathrm{dt}, 1 \mathrm{H}, J=4.5,9.0 \mathrm{~Hz}), 4.97$ (d, 1H, J= 8.1 Hz), 6.67 $(\mathrm{d}, 1 \mathrm{H}, J=8.4 \mathrm{~Hz}), 7.17-7.30(\mathrm{~m}, 5 \mathrm{H}) ;{ }^{13} \mathrm{C} \mathrm{NMR}\left(75 \mathrm{MHz}, \mathrm{CDCl}_{3}\right) \delta$ 21.9, 23.4, 24.2, 24.5, 26.0, 28.2 (3C), 38.1, 42.2, 45.9, 46.3, 49.1, 55.5, 80.0, 126.8, 128.5, 129.4, 136.6, 155.2, 170.2, 170.7.<smiles>O=C(O)C(Cc1ccccc1)NC(=O)N1CCCC1C(=O)N1CCCC1</smiles>

Boc-L-Phe-L-Pro-N $\left(\mathrm{CH}_{2} \mathrm{CH}_{2}\right)_{2}$ : $\quad 79 \%$ yield (white solid) from Boc-L-Pro-OH (liquid-phase synthesis); ${ }^{1} \mathrm{H}$ NMR $\left(500 \mathrm{MHz}, \mathrm{CDCl}_{3}\right) \delta 1.36$ (s, 9H), 1.80-2.06 (m, 6H), 2.08-2.24 $(\mathrm{m}, 2 \mathrm{H}), 2.87(\mathrm{dd}, 1 \mathrm{H}, J=7.5,14.0 \mathrm{~Hz}), 3.14(\mathrm{dd}, 1 \mathrm{H}, J=5.0,13.5 \mathrm{~Hz}), 3.39-3.45(\mathrm{~m}, 2 \mathrm{H})$, $3.47-3.53(\mathrm{~m}, 1 \mathrm{H}), 3.60(\mathrm{ddd}, 1 \mathrm{H}, J=7.0,7.0,12.0 \mathrm{~Hz}), 3.69-3.73(\mathrm{~m}, 1 \mathrm{H}), 3.82(\mathrm{ddd}, 1 \mathrm{H}, J=7.0$, 7.0, $9.0 \mathrm{~Hz}), 5.21(\mathrm{~d}, 1 \mathrm{H}, J=8.5 \mathrm{~Hz}), 4.64-4.72(\mathrm{~m}, 2 \mathrm{H}), 7.21-7.29(\mathrm{~m}, 5 \mathrm{H})$.

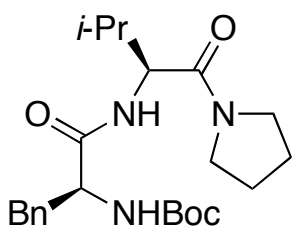

Boc-L-Phe-L-Val-N $\left(\mathrm{CH}_{2} \mathbf{C H}_{2}\right)_{2}$ : $90 \%$ yield (white solid) from Boc-L-Pro-OH (liquid-phase synthesis); ${ }^{1} \mathrm{H}$ NMR $\left(300 \mathrm{MHz}, \mathrm{CDCl}_{3}\right) \delta 0.87(\mathrm{~d}, 3 \mathrm{H}, J=6.6 \mathrm{~Hz}), 0.92(\mathrm{~d}, J=6.6$ Hz), 1.40 (s, 9H), 1.84-2.01 (m, 5H), 3.04-3.13 (m, 2H), 3.37-3.52 (m, 3H), 3.61 (br, 1H), 4.36 (brs, 1H), 4.50 (dd, 1H, $J=7.2,8.7 \mathrm{~Hz}), 4.95(\mathrm{~d}, 1 \mathrm{H}, J=7.8 \mathrm{~Hz}), 6.57$ (d, $J=9.0 \mathrm{~Hz}), 7.17-7.30$ 
$(\mathrm{m}, 5 \mathrm{H})$.

General Procedure for Transforming from Boc-L-Phe-Xxx-N( $\left(\mathrm{CH}_{2} \mathrm{CH}_{2}\right)_{2}$ to Chiral Triamines 1.
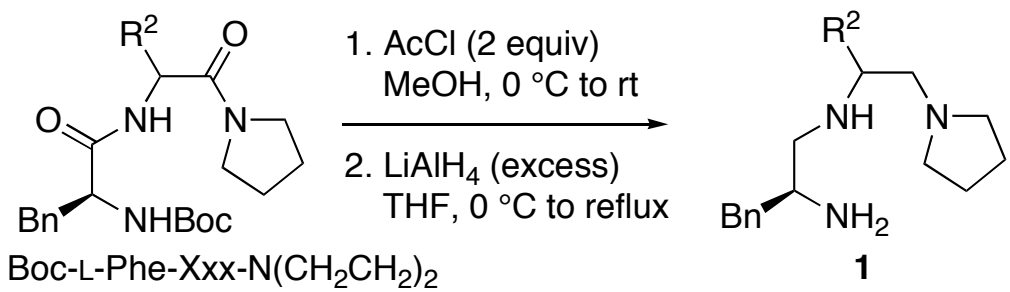

To a solution of Boc-L-Phe- $\mathrm{Xxx}-\mathrm{N}\left(\mathrm{CH}_{2} \mathrm{CH}_{2}\right)_{2}(1 \mathrm{~g})$ in $\mathrm{MeOH}(2 \mathrm{~mL})$ was added dropwise acetyl chloride $(2 \mathrm{~mL})$ at $0{ }^{\circ} \mathrm{C}$. After being stirred for $5 \mathrm{~h}$ at $0{ }^{\circ} \mathrm{C}$, the reaction was allowed to warm to room temperature and stirred for an additional $1 \mathrm{~h}$ and the solution was concentrated in vacuo. To the residual product in THF was added lithium aluminium hydride (13 equiv) at $0{ }^{\circ} \mathrm{C}$. After being stirred for $0.5 \mathrm{~h}$ at $0{ }^{\circ} \mathrm{C}$, the reaction was allowed to heat at reflux for $48 \mathrm{~h}$ before the reaction was quenched with $\mathrm{Na}_{2} \mathrm{SO}_{4}$ and water with vigorous stirring at $0{ }^{\circ} \mathrm{C}$. The white-gray suspension was filtered and concentrated. The crude product was purified by column chromatography on silica gel (Fuji Silysia Chemical Ltd. Cromatorex ${ }^{\circledR}$ NH-DM1020) and ion exchanger (amberlite IRA 458RFCL, eluent: $\mathrm{H}_{2} \mathrm{O}-\mathrm{MeOH}=1: 2$ ) to give triamine 1.<smiles>CC(C)(C)C(CNC(N)Cc1ccccc1)CN1CCCC1</smiles>

(+)-(2S,2S')-2-Amino-1-\{N-[4'-methyl-1'-(pyrrolidin-1'’-yl)pentan-2'-yl]am ino\}-3-phenylpropane (1k): $82 \%$ yield (yellow solid); IR (KBr) 3301, 2953, 1600, 1495, 1462, 1365, 1354, $1140 \mathrm{~cm}^{-1} ;{ }^{1} \mathrm{H}$ NMR (300 MHz, $\left.\mathrm{CDCl}_{3}\right) \delta 0.88$ (d, 3H, J=2.0 Hz), 0.89 (d, 3H, $J=2.0$ $\mathrm{Hz}), 1.16(\mathrm{ddd}, 1 \mathrm{H}, J=6.5,6.5,13.5 \mathrm{~Hz}), 1.31(\mathrm{ddd}, 1 \mathrm{H}, J=5.5,8.0,13.5 \mathrm{~Hz}), 1.68(\mathrm{tt}, 1 \mathrm{H}, J=6.5$, $13.0 \mathrm{~Hz}), 1.74$ (brt, 3H, $J=6.5 \mathrm{~Hz}), 2.26(\mathrm{dd}, 1 \mathrm{H}, J=4.0,12.0 \mathrm{~Hz}), 2.36-2.44(\mathrm{~m}, 2 \mathrm{H}), 2.44-2.58$ (m, 4H), 2.58-2.64 (m, 1H), $2.71(\mathrm{dd}, 1 \mathrm{H}, J=4.5,12.0 \mathrm{~Hz}), 2.78(\mathrm{dd}, 1 \mathrm{H}, J=4.5,13.0 \mathrm{~Hz}), 3.09$ (tt, $1 \mathrm{H}, J=4.0,8.5 \mathrm{~Hz}), 7.21-7.31(\mathrm{~m}, 5 \mathrm{H}) ;{ }^{13} \mathrm{C} \mathrm{NMR}\left(75 \mathrm{MHz}, \mathrm{CDCl}_{3}\right) \delta 22.8,23.3,23.5(2 \mathrm{C}), 25.0$, $42.5,43.1,52.7,53.8,54.1,54.4,61.1,126.1,128.4(2 \mathrm{C}), 129.3(2 \mathrm{C}), 139.4 ;[\alpha]_{\mathrm{D}}{ }^{24.5}=57.7(c 1.00$, $\mathrm{CHCl}_{3}$ ). HRMS (FAB) Calcd for $\mathrm{C}_{19} \mathrm{H}_{33} \mathrm{~N}_{3}[\mathrm{M}+\mathrm{Na}]^{+}:$326.2572, Found 326.2587.<smiles>NC(Cc1ccccc1)CN1CCCC1CN1CCCC1</smiles>

(-)-(2S,2'S)-2-Amino-1-phenyl-3-[2'-(pyrrolidin-1'-ylmethyl)pyrrolidin-1'yl]- 
propane (11): 79\% yield (colorless oil); IR (film) 3026, 2959, 2790, 1739, 1495, 1453, 1364, 1240, $1047 \mathrm{~cm}^{-1} ;{ }^{1} \mathrm{H}$ NMR (300 MHz, $\mathrm{CDCl}_{3}$ ) $\delta 1.49$ (br, 2H), 1.60-1.79 (m, 7H), 1.89-2.11 (m, $1 \mathrm{H}), 2.27-2.58(\mathrm{~m}, 10 \mathrm{H}), 2.74(\mathrm{dd}, 1 \mathrm{H}, J=6.9,12.0 \mathrm{~Hz}), 2.97(\mathrm{dd}, 1 \mathrm{H}, J=3.9,13.2 \mathrm{~Hz}), 3.06-3.17$ $(\mathrm{m}, 2 \mathrm{H}), 7.21-7.33(\mathrm{~m}, 5 \mathrm{H}) ;[\alpha]_{\mathrm{D}}^{21.8}=-42.6\left(c 1.47, \mathrm{CDCl}_{3}\right)$.<smiles>CCCC(CN1CCCC1)NCC(N)Cc1ccccc1</smiles>

(+)-(2S,2'S)-2-Amino-1-\{N-[3'-methyl-1'-(pyrrolidin-1'-yl)butan-2'-yl]ami no\}-3-phenylpropane (1m): $79 \%$ yield (yellow solid); IR (KBr) 3385, 2953, 2817, 1491, 1463, 1356, 1137, $1110 \mathrm{~cm}^{-1} ;{ }^{1} \mathrm{H} \mathrm{NMR}\left(300 \mathrm{MHz}, \mathrm{CDCl}_{3}\right) \delta 0.87$ (d, 3H, $\left.J=6.6\right), 0.89$ (d, 3H, $J=6.6$ ), $1.60-1.90(\mathrm{~m}, 5 \mathrm{H}), 2.18(\mathrm{dd}, 1 \mathrm{H}, J=10.2,18.3 \mathrm{~Hz}), 2.33-2.59(\mathrm{~m}, 9 \mathrm{H}), 2.72(\mathrm{dd}, 1 \mathrm{H}, J=3.9,12.0$ $\mathrm{Hz}), 2.77(\mathrm{dd}, 1 \mathrm{H}, J=4.5,12.9 \mathrm{~Hz}), 3.09(\mathrm{tt}, 1 \mathrm{H}, J=4.2,8.4 \mathrm{~Hz}), 7.21-7.32(\mathrm{~m}, 5 \mathrm{H}) ;{ }^{13} \mathrm{C} \mathrm{NMR}(75$ $\left.\mathrm{MHz}, \mathrm{CDCl}_{3}\right) \delta 16.9,18.8,23.5$ (2C), 28.7, 42.3, 52.7, 54.4, 54.6 (2C), 56.4, 60.9, 126.1, 128.3 (2C), $129.2(2 \mathrm{C}), 139.5 ;[\alpha]_{\mathrm{D}}^{24.2}=62.6$ (c 1.24, $\mathrm{CHCl}_{3}$ ). HRMS (FAB) Calcd for $\mathrm{C}_{18} \mathrm{H}_{31} \mathrm{~N}_{3}$ $[\mathrm{M}+\mathrm{H}]^{+}$290.2596, Found 290.2598.

\section{Preparation of $\alpha$-Acyloxyacrolein. ${ }^{7}$}

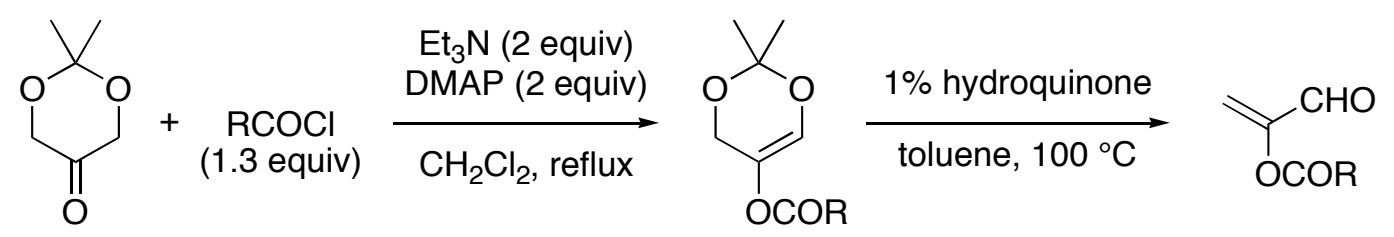

General Procedure for Preparing Acyloxydioxin. ${ }^{4}$ To a solution of 2,2-dimethyl-5-oxo-1,3-dioxane $(0.836 \mathrm{~mL}, 7.0 \mathrm{mmol})$ in $\mathrm{CH}_{2} \mathrm{Cl}_{2}(14 \mathrm{~mL})$ at room temperature were added successively $\mathrm{NEt}_{3}(1.95 \mathrm{~mL}, 14 \mathrm{mmol})$, DMAP (171 mg, $\left.1.4 \mathrm{mmol}\right)$, and acyl chloride $(9.1 \mathrm{mmol})$. The resulting solution was refluxed for $6 \mathrm{~h}$. The reaction mixture was cooled to room temperature and extracted with $\mathrm{Et}_{2} \mathrm{O}$. The combined organic layer was washed with brine, dried over $\mathrm{MgSO}_{4}$ and concentrated in vacuo. Purification of the residue was accomplished by silica gel chromatography using hexane- $\mathrm{Et}_{2} \mathrm{O}$ as the eluent.<smiles>CCCCOC1=COC(C)(C)OC1</smiles>

Bz 2,2-Dimethyl-4H-1,3-dioxin-5-yl benzoate: ${ }^{7} \quad 95 \%$ yield (white solid); ${ }^{1} \mathrm{H}$ NMR (300 MHz, $\left.\mathrm{CDCl}_{3}\right) \delta 1.58(\mathrm{~s}, 6 \mathrm{H}), 4.39(\mathrm{~d}, 2 \mathrm{H}, J=1.5 \mathrm{~Hz}), 6.64(\mathrm{~s}, 1 \mathrm{H}), 7.48(\mathrm{t}, 2 \mathrm{H}, J=7.8 \mathrm{~Hz}), 7.62(\mathrm{t}, 1 \mathrm{H}, J$ $=7.8 \mathrm{~Hz}), 8.07(\mathrm{~d}, 2 \mathrm{H}, J=6.9 \mathrm{~Hz})$. 
OCOC $6 \mathrm{H}_{4}(\mathrm{OMe})^{p}$ 2,2-Dimethyl-4H-1,3-dioxin-5-yl 4-Methoxybenzoate: $92 \%$ yield (white solid); ${ }^{1} \mathrm{H}$ NMR (300 MHz, $\left.\mathrm{CDCl}_{3}\right) \delta 1.57$ (s, 6H), $3.88(\mathrm{~s}, 3 \mathrm{H}), 4.37$ (d, 2H, J = 1.8 Hz), 6.61 (t, $1 \mathrm{H} J=1.5 \mathrm{~Hz}), 6.94(\mathrm{~d}, 2 \mathrm{H}, J=9.0 \mathrm{~Hz}), 8.02(\mathrm{~d}, 2 \mathrm{H}, J=9.0 \mathrm{~Hz})$.<smiles>CC(=O)OC1=COC(C)(C)OC1</smiles>

OAc 2,2-Dimethyl-4H-1,3-dioxin-5-yl Acetate: ${ }^{7} \quad 91 \%$ yield (white solid); ${ }^{1} \mathrm{H}$ NMR $(300 \mathrm{MHz}$, $\left.\mathrm{CDCl}_{3}\right) \delta 1.52(\mathrm{~s}, 6 \mathrm{H}), 2.14(\mathrm{~s}, 3 \mathrm{H}), 4.25(\mathrm{~d}, 2 \mathrm{H}, J=1.5 \mathrm{~Hz}), 6.50(\mathrm{~s}, 1 \mathrm{H})$.

General Procedure for Preparing $\boldsymbol{\alpha}$-Acyloxyacroleins. To toluene $(17 \mathrm{~mL})$ were added 2,2-dimethyl-4H-1,3-dioxin-5-yl acylate $(8.8 \mathrm{mmol})$ and hydroquinone $(380 \mathrm{mg})$. The resulting mixture was heated at $100{ }^{\circ} \mathrm{C}$ for 12 20 hours. The reaction mixture was concentrated in vacuo. Purification of the crude residue on silica gel (eluent: pentane- $\mathrm{Et}_{2} \mathrm{O}$ ) yielded the corresponding $\alpha$-acyloxyacrolein in good yield.<smiles>C=C(C)OC(=O)OCc1ccccc1</smiles>

$\mathrm{CHO}_{\boldsymbol{\alpha}}$-Benzoyloxyacrolein: ${ }^{7} \quad 92 \%$ yield (a white solid); ${ }^{1} \mathrm{H}$ NMR $\left(300 \mathrm{MHz}, \mathrm{CDCl}_{3}\right) \delta 6.06$ $(\mathrm{d}, 1 \mathrm{H}, J=2.4 \mathrm{~Hz}), 6.23$ (d, 1H, $J=2.4 \mathrm{~Hz}), 7.50$ (t, 2H, $J=7.2 \mathrm{~Hz}), 7.64$ (t, 1H, J= 7.2 Hz), 8.14 $(\mathrm{d}, 2 \mathrm{H}, J=7.2 \mathrm{~Hz}), 9.50(\mathrm{~s}, 1 \mathrm{H})$.<smiles>C=C(C=O)OC(=O)OCC(=O)OCc1ccccc1</smiles>
2925, 2857, 1734, 1698, 1605, 1509, 1458, 1320, 1259, 1166, 1089, 1023, 940, 849, 765, $689 \mathrm{~cm}^{-1}$, ${ }^{1} \mathrm{H} \mathrm{NMR}\left(300 \mathrm{MHz}, \mathrm{CDCl}_{3}\right) \delta 3.88(\mathrm{~s}, 3 \mathrm{H}), 6.03(\mathrm{~d}, 1 \mathrm{H}, J=2.4 \mathrm{~Hz}), 6.19(\mathrm{~d}, 1 \mathrm{H}, J=2.4 \mathrm{~Hz}), 6.96$ $(\mathrm{d}, 2 \mathrm{H}, J=9.0 \mathrm{~Hz}), 8.08(\mathrm{~d}, 2 \mathrm{H}, J=9.0 \mathrm{~Hz}), 9.48(\mathrm{~s}, 1 \mathrm{H}) ;{ }^{13} \mathrm{C} \mathrm{NMR}\left(75 \mathrm{MHz}, \mathrm{CDCl}_{3}\right) \delta 55.5,113.9$ (2C), 120.5, 121.3, 132.5 (2C), 152.8, 163.6, 164.2, 185.5. Anal. Calcd for $\mathrm{C}_{11} \mathrm{H}_{10} \mathrm{O}_{4}$ : C, 64.07; H, 4.89; Found: C, 63.91; H, 4.81.<smiles>C=CCCC(=O)O</smiles>

$\mathrm{CHO} \boldsymbol{\alpha}$-Acetyloxyacrolein: ${ }^{7} \quad 70 \%$ yield (colorless oil); ${ }^{1} \mathrm{H}$ NMR $\left(300 \mathrm{MHz}, \mathrm{CDCl}_{3}\right) \delta 2.27$ (s, $3 \mathrm{H}), 5.95(\mathrm{~d}, 1 \mathrm{H}, J=2.4 \mathrm{~Hz}), 6.09(\mathrm{~d}, 1 \mathrm{H}, J=2.4 \mathrm{~Hz}), 9.38(\mathrm{~s}, 1 \mathrm{H})$.

Representative Procedure for the Enantioselective Diels-Alder Reaction. To a solution of $1 \mathbf{k}(24.3 \mathrm{mg}, 0.08 \mathrm{mmol})$ and pentafluorobenzenesulfonic acid $(56.8 \mathrm{mg}, 0.22 \mathrm{mmol})$ in $0.125 \mathrm{~mL}$ of nitroethane or $0.25 \mathrm{~mL}$ of THF was added diene $(1.6 \mathrm{mmol})$. After being stirred at $0{ }^{\circ} \mathrm{C}$ or room temperature, $\alpha$-substituted acrolein $(0.8 \mathrm{mmol})$ was added in one portion. Upon 
consumption of $\alpha$-substituted acrolein, the reaction was quenched with saturated aqueous $\mathrm{NaHCO}_{3}$ and the reaction mixture was diluted with pentane and washed successively with $\mathrm{H}_{2} \mathrm{O}$ and brine. The organic layer was dried $\left(\mathrm{MgSO}_{4}\right)$, filtered, and concentrated under reduced pressure. Purification of the Diels-Alder adduct was accomplished by silica gel chromatography eluting with pentane-ether.

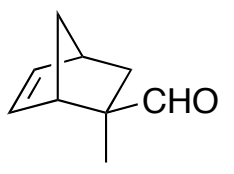

$(1 S, 2 R, 4 S)$-2-Formyl-2-methylbicyclo[2.2.1]hept-5-ene $\quad($ exo isomer $):^{8} \quad$ The exo-endo ratio was determined by ${ }^{1} \mathrm{H}$ NMR analysis: ${ }^{8} \quad{ }^{1} \mathrm{H}$ NMR $\left(300 \mathrm{MHz}, \mathrm{CDCl}_{3}\right) \delta 9.40(\mathrm{~s}, 1 \mathrm{H}$, CHO (endo)), $9.69(\mathrm{~s}, 1 \mathrm{H}, \mathrm{CHO}($ exo $))$. The ee was determined by GC analysis after conversion to chiral acetals by $(-)-(2 R, 4 R)-2,4-$ pentanediol: $\mathrm{GC}\left(\mathrm{PEG}\right.$, column temp. $\left.=80{ }^{\circ} \mathrm{C}\right) t_{\mathrm{R}}=37.7$ (endo-isomer), 47.6 (endo-isomer), 51.5 ((1S,2R,4S)-isomer), $54.7((1 R, 2 S, 4 R)$-isomer) min. The absolute configuration was established by comparison of optical rotation values with data in the literature. ${ }^{8}$<smiles>CC1=C(C)C[C@](C)(O[18O])CC1</smiles>

(-)-(S)-1-Formyl-3,4-dimethylcyclohex-3-enyl Benzoate: White solid; IR (film) 2916, 2841, 1737, 1710, 1601, 1584, 1451, 1383, 1316, 1291, 1247, 1175, 1112, 1068, $1026 \mathrm{~cm}^{-1}$; ${ }^{1} \mathrm{H}$ NMR (300 MHz, $\mathrm{CDCl}_{3}$ ) $\delta 1.67$ (brs, 6H), 1.84 (ddd, 1H, J=6.0, 9.6, $12.9 \mathrm{~Hz}$ ), 2. 01-2.30 (m, $3 \mathrm{H}), 2.37(\mathrm{brd}, 1 \mathrm{H}, J=18.9 \mathrm{~Hz}), 2.71(\mathrm{brd}, 1 \mathrm{H}, J=18.0 \mathrm{~Hz}), 7.46(\mathrm{t}, 2 \mathrm{H}, J=7.8 \mathrm{~Hz}), 7.60(\mathrm{t}, 1 \mathrm{H}, J$ $=7.8 \mathrm{~Hz}), 8.03(\mathrm{~d}, 2 \mathrm{H}, J=7.8 \mathrm{~Hz}), 9.66(\mathrm{~s}, 1 \mathrm{H}) ;{ }^{13} \mathrm{C} \mathrm{NMR}\left(125 \mathrm{MHz}, \mathrm{CDCl}_{3}\right) \delta 18.7,18.8,27.1$, $27.7,35.2,83.8,121.3,124.9,128.5(2 \mathrm{C}), 129.5,129.8(2 \mathrm{C}), 133.5,166.0,198.4 ;[\alpha]_{\mathrm{D}}=-25.9(c$ 1.62, $\mathrm{CHCl}_{3}$ ) for $87 \%$ ee. The ee was determined by chiral HPLC (Daicel Chiralcel OD-H, hexane $: i-\mathrm{PrOH}=200: 1$, flow rate $0.75 \mathrm{~mL} / \mathrm{min}$ ) $t_{\mathrm{R}}=13.2$ (minor) and 15.4 (major) min. The absolute configuration was not established.

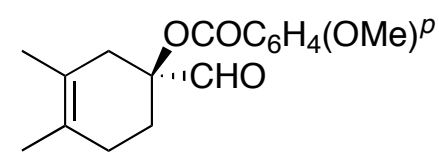

(-)-(S)-1-Formyl-3,4-dimethylcyclohex-3-enyl p-Methoxybenzoate:

white solid; IR (KBr) 3436, 2925, 2853, 1726, 1702, 1604, 1577, 1509, 1421, 1300, 1251, 1166, 1104, 1069, $1022 \mathrm{~cm}^{-1} ;{ }^{1} \mathrm{H}$ NMR $\left(300 \mathrm{MHz}, \mathrm{CDCl}_{3}\right) \delta 1.81$ (ddd, $\left.1 \mathrm{H}, J=5.7,9.6,15.6 \mathrm{~Hz}\right), 1.66$ (brs, 6H), 2.00-2.18 (m, 3H), 2.34 (brd, 1H, $J=18.3 \mathrm{~Hz}$ ), 2.69 (brd, 1H, $J=18.0 \mathrm{~Hz}$ ), 3.87 (s, 3H), $6.94(\mathrm{~d}, \mathrm{~J}=8.7 \mathrm{~Hz}), 7.98(\mathrm{~d}, 2 \mathrm{H}, J=8.7 \mathrm{~Hz}), 9.65(\mathrm{~s}, 1 \mathrm{H}) ;{ }^{13} \mathrm{C} \mathrm{NMR}\left(75 \mathrm{MHz}, \mathrm{CDCl}_{3}\right) \delta 18.7,18.9$, $27.0,27.7,35.2,55.5,83.3,113.7(2 \mathrm{C}), 121.4,121.7,124.8,131.9(2 \mathrm{C}), 163.8,165.7,198.6 ;[\alpha]_{\mathrm{D}}=$ -24.7 (c 1.10, $\mathrm{CHCl}_{3}$ ). Anal. Calcd for $\mathrm{C}_{17} \mathrm{H}_{20} \mathrm{O}_{4}: \mathrm{C}, 70.81 ; \mathrm{H}, 6.99$. Found: C, 70.74; H, 6.88. The ee was determined by HPLC analysis (Daicel Chiralcel OD-H column, hexane- $i$-PrOH $=40$ : 
1; flow rate $=0.5 \mathrm{~mL} / \mathrm{min})$ after conversion to (1-hydroxy-3,4-dimethylcyclohex-3-enyl)methyl benzoate by reducing with $\mathrm{LiAlH}_{4}$ (2 equiv) in THF at room temperature and the subsequent selective benzoylation of primary hydroxy group of the corresponding 1,2-diol with benzoyl chloride (1.3 equiv) in the presence of $N, N$-diisopropylethylamine (2 equiv) in dichloromethane at $-78{ }^{\circ} \mathrm{C}^{9}: t_{\mathrm{R}}=31.2$ (major) and 35.7 (minor) min. The absolute configuration was not established.

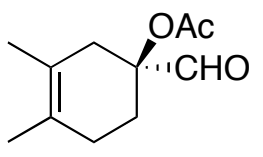

(-)-(S)-1-Formyl-3,4-dimethylcyclohex-3-enyl p-Acetate: Colorless oil; IR (film) 2918, 2860, 2720, 1737, 1371, 1274, 1239, 1130, 1070, $1024 \mathrm{~cm}^{-1}$; ${ }^{1} \mathrm{H}$ NMR (300 MHz, $\left.\mathrm{CDCl}_{3}\right) \delta 1.64$ (brs, 6H), 1.70-1.80 (m, 1H), 1.90-2.08 (m, 3H), $2.11(\mathrm{~s}, 3 \mathrm{H}), 2.20$ (brd, 1H, $J=$ 14.4 Hz), 2.57 (brd, $1 \mathrm{H}, J=18.0 \mathrm{~Hz}), 9.53(\mathrm{~s}, 1 \mathrm{H}) ;{ }^{13} \mathrm{C} \mathrm{NMR}\left(125 \mathrm{MHz}, \mathrm{CDCl}_{3}\right) \delta 18.7,18.8,20.9$, $26.8,27.5,35.4,83.2,121.2,125.0,170.7,198.5 ;[\alpha]_{\mathrm{D}}=-1.0\left(c 1.06, \mathrm{CHCl}_{3}\right)$. The ee was determined by HPLC analysis (Daicel Chiralcel OD-H column, hexane- $i$-PrOH $=40: 1$; flow rate $=0.5 \mathrm{~mL} / \mathrm{min}$ ) after conversion to (1-hydroxy-3,4-dimethylcyclohex-3-enyl)methyl benzoate by reducing with $\mathrm{LiAlH}_{4}$ (2 equiv) in THF at room temperature and the subsequent selective benzoylation of primary hydroxy group of the corresponding 1,2-diol with benzoyl chloride (1.3 equiv) in the presence of $N, N$-diisopropylethylamine (2 equiv) in dichloromethane at $-78{ }^{\circ} \mathrm{C}^{9}: t_{\mathrm{R}}=$ 31.2 (major) and 35.7 (minor) min. The absolute configuration was not established.

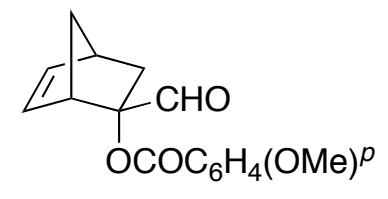

(-)-(1S,2S,4S)-2-Formylbicyclo[2.2.1]hept-5-en-2-yl p-Methoxybenzoate (exo isomer): White solid; IR (KBr) 3067, 2978, 2841, 2720, 1734, 1705, 1607, 1579, 151, 1460, 1421, 1320, 1259, 1166, 1143, 1101, 1069, 1044, $1027 \mathrm{~cm}^{-1} ;{ }^{1} \mathrm{H}$ NMR $\left(300 \mathrm{MHz}, \mathrm{CDCl}_{3}\right) \delta 1.33$ $(\mathrm{dd}, 1 \mathrm{H}, J=3.6,12.6 \mathrm{~Hz}), 1.46-1.51(\mathrm{~m}, 1 \mathrm{H}), 1.76(\mathrm{br}, 1 \mathrm{H}) ; 2.64(\mathrm{dd}, 1 \mathrm{H}, J=3.6,13.2 \mathrm{~Hz}), 3.01$ (br, 1H), 3.26 (br, 1H), 3.87 (s, 3H), $6.22(\mathrm{dd}, 1 \mathrm{H}, J=3.0,5.7 \mathrm{~Hz}), 6.47$ (dd, 1H, J = 3.0, $5.4 \mathrm{~Hz}$ ), $6.93(\mathrm{~d}, 2 \mathrm{H}, J=8.7 \mathrm{~Hz}), 7.94(\mathrm{~d}, 2 \mathrm{H}, J=9.0 \mathrm{~Hz}), 9.74(\mathrm{~s}, 1 \mathrm{H}) ;{ }^{13} \mathrm{C} \mathrm{NMR}\left(75 \mathrm{MHz}, \mathrm{CDCl}_{3}\right) \delta 38.0$, 42.3, 45.6, 48.7, 55.5, 91.6, 113.7, 121.5, 131.9, 132.4, 140.8 (2C), 163.8 (2C), 166.3, 198.8; $[\alpha]_{\mathrm{D}}^{22.3}=-104.4\left(c\right.$ 1.02, $\left.\mathrm{CHCl}_{3}\right)$. The exo-endo ratio was determined by ${ }^{1} \mathrm{H} \mathrm{NMR}$ analysis: $\delta 9.53$ (s, 1H, CHO (endo-isomer)) and 9.74 (s, 1H, CHO (exo-isomer)). The relative stereochemistry of major diastereomer was established by comparison with ${ }^{1} \mathrm{H}$ NMR spectral data in the literature ${ }^{10}$ after conversion to the corresponding diol by reducing with $\mathrm{LiAlH}_{4}$ (2 equiv) in THF at room temperature. The ee was determined by HPLC analysis (Daicel Chiralcel OJ-H column, hexane- $i-\mathrm{PrOH}=40: 1$; flow rate $=1 \mathrm{~mL} / \mathrm{min}$ ) after conversion to (2-hydroxybicyclo[2.2.1] hept-5-en-2-yl)methyl benzoate by reducing with $\mathrm{LiAlH}_{4}$ (2 equiv) in THF at room temperature and the subsequent selective benzoylation of primary hydroxy group of the 
corresponding 1,2-diol with benzoyl chloride (1.3 equiv) in the presence of $N, N$-diisopropylethylamine (2 equiv) in dichloromethane at $-78{ }^{\circ} \mathrm{C}^{9}: \quad t_{\mathrm{R}}=15.9$ (minor endo-enantiomer), 17.6 (major endo-enantiomer), 20.9 (minor exo-enantiomer), and 23.4 (major exo-enantiomer) min. The absolute configuration was established by comparison with optical rotation values with data in the literature ${ }^{10}$ after conversion to (-)-(1S,4S)-bicyclo[2.2.1] hept-5-en-2-one by reducing with $\mathrm{LiAlH}_{4}$ (2 equiv) in THF at room temperature and the subsequent oxidative cleavage of the corresponding 1,2-diol with $\mathrm{NaIO}_{4}(1.0$ equiv) in the presence of $\mathrm{KH}_{2} \mathrm{PO}_{4}\left(1\right.$ equiv) in THF- $\mathrm{H}_{2} \mathrm{O}(1: 1 \mathrm{v} / \mathrm{v})$ at room temperature.

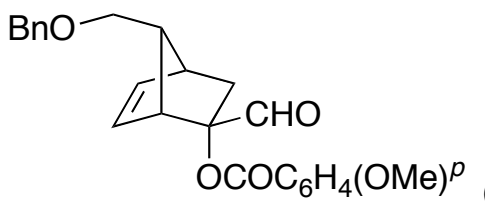

(-)-(1R,2S,4S,7R)-7-Benzyloxymethyl-2-formylbicyclo[2.2.1] hept-5en-2-yl p-Methoxybenzoate (exo isomer): $\quad$ white solid; IR (KBr) 3063, 2943, 2843, 1733, 1705, 1606, 1580, 1511, 1455, 1421, 1320, 1259, 1168, 1100, $1028 \mathrm{~cm}^{-1} ;{ }^{1} \mathrm{H}$ NMR $\left(300 \mathrm{MHz}, \mathrm{CDCl}_{3}\right) \delta$ $1.38(\mathrm{~d}, 1 \mathrm{H}, J=13.2 \mathrm{~Hz}), 2.62(\mathrm{t}, 1 \mathrm{H}, J=7.2 \mathrm{~Hz}), 2.72(\mathrm{dd}, 1 \mathrm{H}, J=3.6,13.2 \mathrm{~Hz}), 2.97(\mathrm{br}, 1 \mathrm{H})$, $3.27(\mathrm{br}, 1 \mathrm{H}), 3.83(\mathrm{dd}, 2 \mathrm{H}, J=0.9,6.9 \mathrm{~Hz}), 3.86(\mathrm{~s}, 3 \mathrm{H}), 4.43(\mathrm{~s}, 2 \mathrm{H}), 6.08(\mathrm{dd}, J=3.0,5.7 \mathrm{~Hz})$, $6.32(\mathrm{dd}, 1 \mathrm{H}, J=3.0,5.7 \mathrm{~Hz}), 6.92(\mathrm{~d}, 2 \mathrm{H}, J=9.0 \mathrm{~Hz}), 7.92(\mathrm{~d}, 2 \mathrm{H}, J=9.0 \mathrm{~Hz}), 9.72(\mathrm{~s}, 1 \mathrm{H})$; $[\alpha]_{\mathrm{D}}^{22.7}=-30.0\left(c 1.49, \mathrm{CHCl}_{3}\right)$ for $74 \%$ ee. The exo-endo ratio was determined by ${ }^{1} \mathrm{H}$ NMR analysis: $\quad \delta 9.54(\mathrm{~s}, 1 \mathrm{H}, \mathrm{CHO}($ endo $))$ and $9.73(\mathrm{~s}, 1 \mathrm{H}, \mathrm{CHO}($ exo $))$. The relative stereochemistry of major diastereomer was established by comparison with ${ }^{1} \mathrm{H}$ NMR spectral data in the literature ${ }^{11}$ after conversion to the corresponding diol by reducing with $\mathrm{LiAlH}_{4}$ (2 equiv) in THF at room temperature. The ee was determined by HPLC analysis (two Daicel Chiralpak AD-H columns; hexane- $i-\mathrm{PrOH}=40: 1$; flow rate $=1 \mathrm{~mL} / \mathrm{min}$ ): $t_{\mathrm{R}}=55.0$ (major exo-enantiomer) and 68.9 (minor exo-enantiomer) min. The absolute configuration was established by comparison with optical rotation values with data in the literature ${ }^{11}$ after conversion to 7-(benzyloxymethyl)-2-(hydroxymethyl)bicyclo[2.2.1] hept-5-en-2-ol by reducing with $\mathrm{LiAlH}_{4}$ (2 equiv) in THF at room temperature

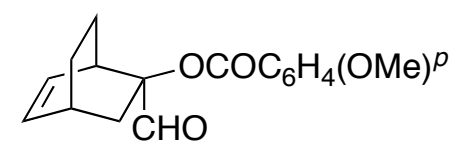

(+)-(1R,2R,4R)-2-Formylbicyclo[2.2.2]oct-5-en-2-yl

p-Methoxybenzoate (endo isomer): white solid; IR (KBr) 3051, 2946, 2870, 2841, 1739, 1704, 1606, 1580, 1511, 1463, 1421, 1370, 1319, 1259, 1227, 1169, 1101, 1070, $1029 \mathrm{~cm}^{-1}$; ${ }^{1} \mathrm{H}$ NMR $\left(300 \mathrm{MHz}, \mathrm{CDCl}_{3}\right) \delta 1.20-1.46(\mathrm{~m}, 2 \mathrm{H}), 1.56-1.73(\mathrm{~m}, 2 \mathrm{H}), 2.25(\mathrm{tt}, 1 \mathrm{H}, J=3.0,12.3 \mathrm{~Hz}), 2.41(\mathrm{td}$, $1 \mathrm{H}, J=3.0,14.4 \mathrm{~Hz}), 2.79(\mathrm{br}, 1 \mathrm{H}), 2.96-3.04(\mathrm{~m}, 1 \mathrm{H}), 3.88(\mathrm{~s}, 3 \mathrm{H}), 6.07$ (t, 1H, $J=7.5 \mathrm{~Hz}), 6.44$ $(\mathrm{t}, 1 \mathrm{H}, J=7.5 \mathrm{~Hz}) 6.95(\mathrm{~d}, 2 \mathrm{H}, J=9.0 \mathrm{~Hz}), 8.01(\mathrm{~d}, 2 \mathrm{H}, J=9.0 \mathrm{~Hz}), 9.43(\mathrm{~s}, 1 \mathrm{H}) ;{ }^{13} \mathrm{C} \mathrm{NMR}(125$ $\left.\mathrm{MHz}_{\mathrm{CDCl}}\right) \delta 19.8,23.9,30.0,35.1,35.4,55.5,85.7,113.8$ (2C), 121.8, 128.9, 131.9 (2C), 137.2, 
163.8, 165.9, 197.8; $[\alpha]_{\mathrm{D}}^{22.6}=7.6\left(c 1.00, \mathrm{CHCl}_{3}\right)$ for $91 \%$ ee. The exo-endo ratio was determined by ${ }^{1} \mathrm{H}$ NMR analysis: $\delta 9.43(\mathrm{~s}, 1 \mathrm{H}, \mathrm{CHO}$ (endo)) and $9.52(\mathrm{~s}, 1 \mathrm{H}, \mathrm{CHO}($ exo $)$ ). The relative stereochemistry of major diastereomer was established by X-ray crystallographic analysis of benzoate analogue (see below). The ee was determined by HPLC analysis (Daicel Chiralcel OD-H column; hexane- $i$ - $\mathrm{PrOH}=40: 1$; flow rate $=1 \mathrm{~mL} / \mathrm{min}$ ) after conversion to (2-hydroxybicyclo[2.2.2] oct-5-en-2-yl)methyl benzoate by reducing with $\mathrm{LiAlH}_{4}$ (2 equiv) in THF at room temperature and the subsequent selective benzoylation of primary hydroxy group of the corresponding 1,2-diol with benzoyl chloride $(1.3$ equiv) in the presence of $N, N$-diisopropylethylamine (2 equiv) in dichloromethane at $-78{ }^{\circ} \mathrm{C}^{9}: \quad t_{\mathrm{R}}=13.6$ (minor endo-enantiomer) and 16.9 (major endo-enantiomer) min. The absolute configuration was established by comparison with optical rotation values with data in the literature ${ }^{12}$ after conversion to $(+)-(1 R, 4 R)$-bicyclo[2.2.2] oct-5-en-2-one by reducing with $\mathrm{LiAlH}_{4}$ (2 equiv) in THF at room temperature and the subsequent oxidative cleavage of the corresponding 1,2-diol with $\mathrm{NaIO}_{4}(1.0$ equiv) in the presence of $\mathrm{KH}_{2} \mathrm{PO}_{4}$ (1 equiv) in THF- $\mathrm{H}_{2} \mathrm{O}(1: 1 \mathrm{v} / \mathrm{v})$ at room temperature.

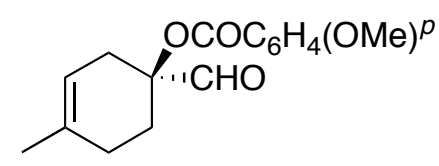

(-)-1-Formyl-4-methylcyclohex-3-enyl p-Methoxybenzoate: white solid; IR (KBr) 3013, 2914, 1844, 1726, 1696, 1604, 1577, 1508, 1453, 1428, 1387, 1303, 1255 , 1167, 1104, 1070, $1023 \mathrm{~cm}^{-1}$; ${ }^{1} \mathrm{H}$ NMR (300 MHz, $\mathrm{CDCl}_{3}$ ) $\delta 1.72$ (brs, 3H), 1.81-1.94 (m, 1H), 2.00-2.14 (m, 1H), 2.14-2.31 (m, 2H), 2.45 (brd, 1H, J=18.3 Hz), 2.69 (brd, 1H, $J=18.3 \mathrm{~Hz}$ ), $3.87(\mathrm{~s}, 3 \mathrm{H}), 5.35(\mathrm{br}, 1 \mathrm{H}), 6.93(\mathrm{~d}, 2 \mathrm{H}, J=9.0 \mathrm{~Hz}), 7.98(\mathrm{~s}, 2 \mathrm{H}, J=9.0 \mathrm{~Hz}), 9.68(\mathrm{~s}, 1 \mathrm{H}) ;{ }^{13} \mathrm{C} \mathrm{NMR}$ $\left(75 \mathrm{MHz}, \mathrm{CDCl}_{3}\right) \delta 23.2,26.2,26.7,29.6,54.5,113.7$ (2C), 116.5, 121.6, 131.9 (2C), 133.2, 163.8, 165.7, 198.7; $[\alpha]_{D}^{23.7}=-57.0\left(c\right.$ 1.05, $\left.\mathrm{CHCl}_{3}\right)$. Anal. Calcd for $\mathrm{C}_{16} \mathrm{H}_{18} \mathrm{O}_{4}: \quad \mathrm{C}, 70.06 ; \mathrm{H}, 6.61$. Found: C, 69.87; H, 6.63. The ee was determined by HPLC analysis (Daicel Chiralpak AD-H column, hexane- $i-\mathrm{PrOH}=40: 1$; flow rate $=1 \mathrm{~mL} / \mathrm{min}$ ) after conversion to (1-hydroxy-4-methylcyclohex-3-enyl)methyl benzoate by reducing with $\mathrm{LiAlH}_{4}$ (2 equiv) in $\mathrm{THF}$ at room temperature and the subsequent selective benzoylation of primary hydroxy group of the corresponding 1,2-diol with benzoyl chloride $(1.3$ equiv) in the presence of $N, N$-diisopropylethylamine ( 2 equiv) in dichloromethane at $-78{ }^{\circ} \mathrm{C}: \quad t_{\mathrm{R}}=37.8$ (major enantiomer) and 42.7 (minor enantiomer) min. The absolute configuration was not established.

\section{X-ray Crystallographic Analysis of ( \pm )-endo-2-Formylbicyclo[2.2.2]oct-5-en-2-yl Benzoate:}

Crystal data: $\mathrm{C}_{16} \mathrm{H}_{16} \mathrm{O}_{3}, M=256.29$, crystal dimensions $0.60 \times 0.40 \times 0.40 \mathrm{~mm}^{3}$, monoclinic, space group $P 2_{1}(\# 14), a=11.063(7), b=13.580(8), c=9.289(5) \AA, V=1342.5(14) \AA^{3}, Z=4, D_{c}=1.268$ $\mathrm{g} / \mathrm{cm}^{3}, \mu=0.087 \mathrm{~mm}^{-1}, T=223 \mathrm{~K}$. X-ray crystallographic analysis was performed with a Bruker 
SMART APEX diffractometer (graphite monochromator, MoK $\alpha$ radiation, $\lambda=0.71073 \AA$ ). The structure was solved by direct methods and expanded using Fourier techniques. 3535 reflections were independent and unique, and 236 with $I>2 \sigma(I)\left(2 \theta_{\max }=29.21^{\circ}\right)$ were used for the solution of the structure. $R=0.0417$ and $R w=0.115$.

Crystallographic data have been deposited with the Cambridge Crystallographic Data Centre: Deposition number CCDC-272294. Copies of the data can be obtained free of charge via http//www.ccdc.cam.ac.uk/conts/retrieving.html (or from the Cambridge Crystallographic Data Centre, 12, Union Road, Cambridge, CB2 1EZ, UK; Fax: +44 1223 336033; e-mail: deposit@ccdc.cam.ac.uk).

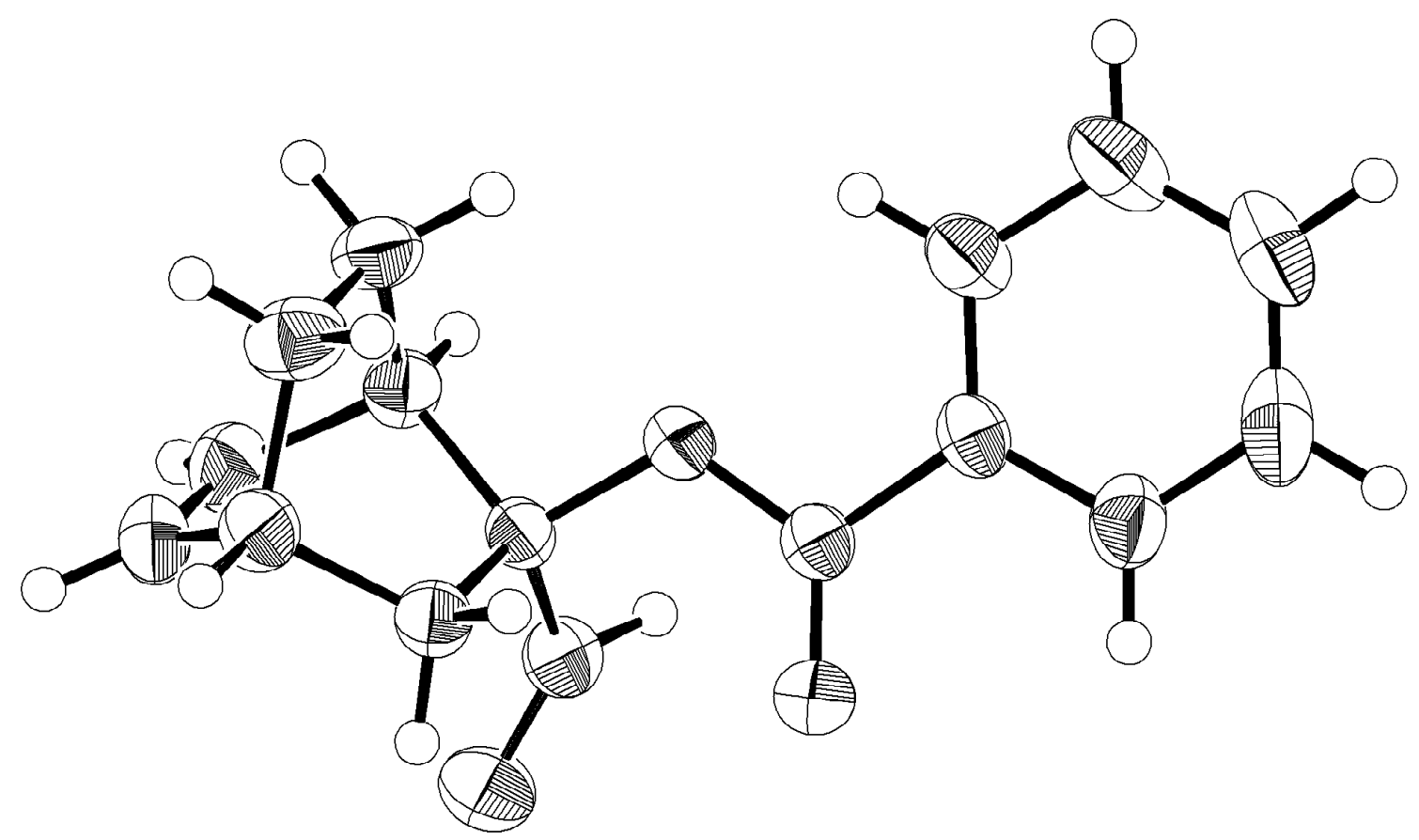

\section{References}

(1) Ager, D. J.; Prakash, I. Synth. Commun. 1996, 26, 3865.

(2) Cantel, S.; Heitz, A.; Martinez, J.; Fehrentz, J.-A. J. Peptide Sci. 2004, 10, 531.

(3) Sato, H. Jpn. Kokai Tokkyo Koho 1999, Patent No. JP 11292832.

(4) Katritzky, A. R.; He, H.-Y.; Verma, A. K. Tetrahedron: Asym. 2002, 13, 933.

(5) Nakadai, M.; Saito, S.; Yamamoto, H. Tetrahedron 2002, 58, 8167.

(6) Lashuel, H. A.; LaBrenz, S. R.; Woo, L.; Serpell, L. C.; Kelly, J. W. J Am. Chem. Soc. 2000, $122,5262$.

(7) Funk, R. L.; Yost, K. J., III J. Org. Chem. 1996, 61, 2598.

(8) (a) Furuta, K.; Shimizu, S.; Miwa, Y.; Yamamoto, H. J. Org. Chem. 1989, 54, 1481.

Furuta, K.; Gao, Q.; Yamamoto, H. Org. Synth. 1995, 72, 86.

(9) Ishihara, K.; Kurihara, H.; Yamamoto, H. J. Org. Chem. 1993, 58, 3791. 
(10) Helmchen, G.; Krotz, A.; Neumánn, H.-P.; Ziegler, M. L. Liebigs Ann. Chem. 1993, 1313.

(11) (a) Corey, E. J.; Loh, T.-P. J. Am. Chem. Soc. 1991, 113, 8966. (b) Corey, E. J.; Loh, T.-P. Tetrahedron Lett. 1993, 34, 3979.

(12) Demuth, M.; Chandrasekhar, S.; Schaffner, K. J. Am. Chem. Soc. 1984, 106, 1092. 